\title{
Discontinuing Monoclonal Antibodies Targeting CGRP Pathway After One-Year Treatment: An Observational Longitudinal Cohort Study
}

Fabrizio Vernieri ( $\square$ f.vernieri@unicampus.it)

Policlinico Universitario Campus Bio-Medico https://orcid.org/0000-0002-9594-9336

Nicoletta Brunelli

Policlinico Universitario Campus Bio-Medico

Roberta Messina

HSR: IRCCS Ospedale San Raffaele

Carmelina Maria Costa

Policlinico Universitario Campus Bio-Medico

\section{Bruno Colombo}

HSR: IRCCS Ospedale San Raffaele

\section{Paola Torelli}

Universita degli Studi di Parma

\section{Simone Quintana}

Azienda Ospedaliero-Universitaria di Parma

\section{Sabina Cevoli}

IRCCS Istituto Delle Scienze Neurologiche di Bologna

\section{Valentina Favoni}

IRCCS Istituto Delle Scienze Neurologiche di Bologna

Florindo d'Onofrio

San Giuseppe Moscati Hospital, Avellino

\section{Gabriella Egeo}

: Sanofi Romania

\section{Renata Rao}

Università di Brescia

\section{Massimo Filippi}

Universita Vita e Salute San Raffaele: Universita Vita Salute San Raffaele

\section{Piero Barbanti}

IRCCS Ospedale San Raffaele Roma

\section{Claudia Altamura}

Policlinico Universitario Campus Bio-Medico 


\section{Research article}

Keywords: calcitonin gene-related peptide, monoclonal antibodies, migraine treatment, real-world, discontinuation

Posted Date: November 10th, 2021

DOI: https://doi.org/10.21203/rs.3.rs-1047534/v1

License: (c) (i) This work is licensed under a Creative Commons Attribution 4.0 International License. Read Full License

Version of Record: A version of this preprint was published at The Journal of Headache and Pain on December 1st, 2021. See the published version at https://doi.org/10.1186/s10194-021-01363-y. 


\section{Abstract}

Background: The monoclonal antibodies anti-calcitonin gene-related peptide (mAbs anti-CGRP) pathway revolutionized migraine prevention. However, some drug agencies limited the treatment to one year due to their high costs. This study aimed at evaluating the effect of discontinuing mAbs anti-CGRP on monthly migraine days (MMDs) and disability in high-frequency episodic (HFEM) and chronic migraine (CM) patients.

Methods: This observational longitudinal cohort study was conducted at 10 Italian headache centres (November 2019-July 2021). Consecutive adult patients followed up for three months (F-UP1-3) after discontinuation of a one-year erenumab/galcanezumab treatment were enrolled. The primary endpoint was the change in F-UP MMDs. Secondary endpoints included variation in pain intensity (Numerical Rating Scale, NRS), monthly painkiller intake (MPI), and HIT-6 scores. We also assessed from F-UP1 to 3 the $\geq 50 \%$ response rates (RR), relapse rate to $\mathrm{CM}$, and recurrence of Medication Overuse (MO).

Results: We enrolled 154 patients ( $72.1 \%$ female, $48.2 \pm 11.1$ years, 107 CM, 47 HFEM); 91 were treated with erenumab, 63 with galcanezumab. From F-UP1 to F-UP3, MMDs, MPI, NRS, and HIT-6 progressively increased but were still lower at F-UP 3 than baseline (Friedman's analysis of rank, p<.001). In the F-UP 13 visits, $\geq 50 \%$ RR frequency did not differ significantly between CM and HFEM patients. However, the median reduction in RR at F-UP3 was higher in HFEM $(-47.7 \%$ [25th, $-79.5 ; 75$ th , -17.0$])$ than in CM patients $(-25.5 \%$ [25th, -47.1 ; 75th ,-3.3]; Mann-Whitney $U$ test; $p=.032)$. Of the 84 baseline CM patients who had reverted to EM, 28 (33.3\%) relapsed to CM at F-UP1, 35 (41.7)\% at F-UP2, 39 (46.4\%) at F-UP3. Of the 64 baseline MO patients ceasing MO, 15 (18.3\%) relapsed to MO at F-UP1, 26 (31.6\%) at F-UP2, and 30 (42.3\%, 11 missing data) at F-UP3. Lower MMDs, MPI, NRS, and HIT-6 and higher RR in the last month of therapy characterized patients with 50\% RR at F-UP1 and F-UP3 (Mann-Whitney U test; consistently $\mathrm{p}<.01)$.

Conclusion: Migraine frequency and disability gradually increased after anti-CGRP mAbs interruption. Most patients did not relapse to MO or CM despite the increase in MMDs. Our data suggest to reconsider mAbs anti-CGRP discontinuation.

\section{Background}

Monoclonal antibodies targeting the calcitonin gene-related peptide (CGRP), i.e., mAbs anti-CGRP pathway, opened a new era in migraine prevention ${ }^{1}$. Randomized clinical trials (RCTs) demonstrated excellent effectiveness and safety of these treatments ${ }^{2-7}$, and increasing real-world studies have confirmed this evidence in real life ${ }^{8,9}$. International drug agencies have approved mAbs anti-CGRP to prevent episodic and chronic migraine in adults since 2018. However, because of the high costs of this therapeutic approach, national institutions set their own rules for their reimbursement to limit expenses. In Italy, anti-CGRP mAbs were initially administered on direct hospital dispensation in episodic (mostly high-frequency episodic migraine, HFEM, i.e., 8-14 monthly migraine days, MMDs) and chronic (1.3 
ICHD- $3^{10}$ ) migraine (CM) patients, until the Italian Medicines Agency (AIFA) defined reimbursement criteria. Since July 2020, reimbursement criteria posed by AIFA to be respected also included a MIDAS score $\geq 11$ when starting mAbs anti-CGRP and a mandatory improvement of that score of at least $50 \%$ after three months to continue. Moreover, Italian authorities currently allow treating both episodic and chronic patients for a maximum of one year, followed by a drug withdrawal of at least 3 months (reduced to one month on July $31^{\text {st }} 2021$ ). However, these reimbursement rules were set after Drug Agencies' approval and before the real-world evidence.

Beyond limiting expenses and regulatory rules about discontinuing mAbs anti CGRP treatment, another important matter is whether those new drugs could modify the history of migraine disease.

Nowadays, we can learn from the real-life experience in the last two years prescribing CGRP targeted mAbs to refine treatment strategies. In particular, we can now provide evidence on the treatment discontinuation after one year. Is this approach correct? If so, how long should this discontinuation last? Should it be applied likewise to episodic and chronic patients? From a different point of view, discontinuing the mAbs anti-CGRP pathway is essential to understand if and to which extent these drugs can be disease-modifying. The study aimed to evaluate the effect of stopping anti-CGRP monoclonal antibodies on monthly migraine days and disability after one year of treatment in HFEM and CM patients.

\section{Methods}

This observational longitudinal cohort study was conducted as part of two observational longitudinal multicentric studies on the real-life use of erenumab (the EARLY-ErenumAb in Real Life in ItalY-study) ${ }^{8}$ and galcanezumab (the GARLIT-GalcanezumAb in Real Life in ITaly-study) ${ }^{9}$, in 10 third level headache centres in Italy.

Consecutive patients diagnosed with HFEM or $\mathrm{CM}$, not previously involved in any randomized clinical CGRP mAbs trial, treated with mAbs anti-CGRP (erenumab or galcanezumab) from November 2019 to July 2021 , according to clinical indication ${ }^{11,12}$, were considered. Data collection of the EARLY and GARLIT studies is described elsewhere ${ }^{8,9}$. The present paper considered the 12-month treatment and 3-month suspension interval regarding erenumab and galcanezumab patients' data from the treatment phase on direct hospital dispensation. All enrolled patients discontinued the drugs for 90 days after one year of treatment, irrespective of the taken $\mathrm{mAb}$.

In brief, monthly migraine days (MMDs), monthly painkiller intake (MPI), monthly disability scale (i.e., Headache Impact Test- HIT-6, Italian version $\left.1.1^{13}\right)$, and pain intensity (0-10 Numerical Rating Scale, NRS) of the monthly most painful attack were prospectively collected. All patients were educated on the headache diary use before enrolment in the EARLY and GARLIT studies. We included in the present study only patients who completed one-year mAbs anti-CGRP treatment followed by a 3-month suspension interval. During the 3-month suspension interval, patients attended clinical visits or were required to send 
monthly a copy of their headache diary (including painkiller intake and pain intensity) and HIT-6 scores by email.

The primary endpoint was to observe the change in MMDs in the three months following mAbs anti-CGRP discontinuation (F-UP 1-2-3) after one year of treatment compared with baseline and last month of mAbs treatment. Moreover, the changes in MMDs for each month in the follow-up period were also assessed as response rates (RR, i.e., percentual reduction) compared to the pre-treatment period (baseline). We calculated the change in RR as the absolute difference to the baseline from the last month of treatment to the $3^{\text {rd }}$ month of follow-up. We also assessed the proportion of patients who could be still be classified as $50 \%$ responders at the follow-up evaluations. Secondary endpoints included changes in MPI, in NRS, and HIT-6 score according to the same intervals. Besides, medication overuse (MO) was also considered as the proportion of patients taking $\geq 15$ NSAIDs or 10 triptans monthly. Among CM patients having episodic migraine at the end of the one-year treatment, we determined the proportion of patients presenting at 15 or more MMDs again at the follow-up evaluations. Finally, we observed the proportion of patients with at least 8 MMDs along evaluation times.

All patients provided written informed consent. The EARLY study received approval no.19/26 from the IRCCS San Raffaele Pisana Institutional Review Board, while the GARLIT study was approved by the Campus Bio-Medico University Ethical Committee n.30/20. The other local Institutional Review Boards mutually recognized the approvals. The GARLIT study has been registered at the Italian Medicines Agency (Agenzia Italiana del Farmaco, AIFA) and at ClinicalTrials.gov NCT04803513.

Anonymized data will be shared by request from any qualified investigator.

Statistical Analysis

Statistical analyses were performed with SPSS version 26.0 (SPSS Inc., Chicago, IL, USA).

This is a priori analysis. The sample size was considered in line with previous studies on the topic. ${ }^{14-18}$ To achieve a power of $80 \%$ and a level of significance of $5 \%$ (two-sided), for detecting an effect size of 0.25 between paired variables, we calculated a sample size of at least 128 subjects. Interval variables were compared between groups with independent t-test (expressed as means with standard deviations [SD]) or Mann-Whitney tests (medians with $25^{\text {th }}, 75^{\text {th }}$ percentiles) according to the results of the Kolmogorov-Smirnov test for data distribution. Friedman's analysis of rank was adopted to analyse the variable changes over time. We described proportions as percentages and categorical variables as frequencies. Contingency tables (Chi-square and two-tailed Fisher exact tests) were run to compare frequencies between groups. Statistical significance was set as two-tailed $p<0.05$. Subjects with missing information regarding the main studied variables (MMDs, NRS, MPI) were excluded. For the other variables, data availability has been declared.

\section{Results}


Among patients enrolled in the EARLY and GARLIT studies, 154 patients ( $72.1 \%$ female, aged $48.2 \pm 11.1$ yrs, min-max 19-71 yrs) respected inclusion criteria. Of these, 107 patients were affected by $\mathrm{CM}$ and 47 by HFEM; 81 patients (69.2\%) presented medication overuse (MO); 91 patients had been treated with erenumab and 63 with galcanezumab. The MMDs, NRS, and MPI were available in all patients during the evaluation times, while HIT-6 scores were fully available in 71 patients (20 with HFEM and $51 \mathrm{CM}$ ).

Table 1 summarizes demographical profiles and clinical variables evaluated at baseline, in the last month of treatment, and follow-up visits in CM and HFEM patients.

After the three-month discontinuation (Table 1), F-UP3 MMDs, MPI, NRS, and HIT-6 were still lower than baseline (Friedman's analysis of rank; consistently, p<.001).

Figure 1 shows the percentage of $\geq 50 \%$ RR patients in CM and HFEM groups in the last month of therapy and at F-UP1-3 visits. No difference was observed in $\geq 50 \%$ RR frequency between CM and HFEM patients along the 3 evaluation times ( $x 2$; consistently $p>.100)$.

Figure 2 shows the percentage of patients having at least 8 MMDs in CM and HFEM groups. No significant difference was observed between $\mathrm{CM}$ and HFEM patients, although the comparison for the first month of follow-up was nearly significant (Mann-Whitney U test; F-UP1: $p=.054$; F-UP2: $p=.692$; FUP3: $p=.144)$.

Table 2 compares the assessed variables according to long-lasting $50 \%$ RR after one (F-UP1) and three (F-UP3) months of discontinuation. As evidenced, patients experiencing a long-lasting F-UP1 and F-UP3 response had lower MMDs, MPI, NRS, and HIT-6 and higher RR in the last month of treatment compared with non-long-lasting responders (Mann-Whitney U test, consistently $p<.001$ ). On the contrary, baseline characteristics, including the type of monoclonal antibody, did not differentiate the responder and nonresponder groups at F-UP1 and F-UP3. Figure 3 shows the median value $(95 \% \mathrm{Cl}$ bars) of MMDs percentual reduction (RR) in the last month of therapy in patients relapsing to $<50 \% R R$ response at F-UP1, F-UP2, F-UP3, and patients still responding at F-UP3 (Mann-Whitney U test). At the end of -year treatment, 84 (78.5\%) baseline CM patients presented episodic migraine, 64 (79.0\%) patients had discontinued MO. Figure 4 displays the rate of relapse to $\mathrm{CM}(\mathrm{A})$ and $\mathrm{MO}(\mathrm{B})$ in these groups of patients.

\section{Discussion}

In the present study, we aimed at evaluating the effect of discontinuation of mAbs anti-CGRP (erenumab and galcanezumab) after 12 months of therapy on monthly migraine days, symptomatic drugs intake, pain severity, and disability in high-frequency migraine and chronic patients. While several RCTs and reallife studies have provided consistent data on the efficacy of this therapy, several questions have yet to be solved regarding its long-term effect on disease history and the rapidity of efficacy vanishing.

Our study demonstrated that MMDs and other considered parameters increased gradually and consistently during the 3 months after discontinuation, both in HFEM and CM patients. Accordingly, 50\% 
RR decreased during 3-month discontinuation in both groups. The wearing-off seemed larger in HFEM patients, which experienced a reduction in $50 \%$ RR from $73.3 \%$ at the 12 th month of therapy to $27.6 \%$ after three months of discontinuation (figure 1). In the CM group, it gradually diminished from $60.6 \%$ to $35.5 \%$ at the end of the third month of suspension. On the other side, $71,4 \%$ of $\mathrm{CM}$ patients presented at least 8 MMDs already at F-UP1, making them again clinically eligible for the treatment, while the same percentage was observed in HFEM patients only at F-UP3.

Randomized clinical trials have poorly addressed the issue of mAbs discontinuation. A gradual reduction of effect on MMDs change after stopping galcanezumab was found in a review of data from the 3 randomized placebo-controlled trials ${ }^{19}$. However, the 4 months of discontinuation followed different treatment periods: a 6-month double-blind period in EM patients and a 3-month double-blind plus a 9month open-label extension period in CM patients.

The effect of discontinuing mAbs anti-CGRP was evaluated in different studies considering small samples of patients ${ }^{15-18}$, mostly treated with erenumab. In each of those studies, MMDs and acute medications intake gradually increased after stopping treatment compared with the last month of mAbs anti-CGRP therapy, in the early phase (4 weeks) ${ }^{16}$ and after three months of evaluation $15,17,18$.

In the study by Gantenbein et al. ${ }^{18}$, MMDs were still reduced in the third month after the last dose only in a small proportion (25\%) of patients. The authors stated that the therapeutic effect of anti-CGRP antibodies outlasting their pharmacological effect is very limited.

More recently, Raffaelli et al. ${ }^{14}$ confirmed that the cessation of mAbs targeting the CGRP pathway determines an increase in migraine frequency and acute medication intake in patients treated with mAbs targeting CGRP receptor (erenumab) or ligand (fremanezumab and galcanezumab). Moreover, the authors observed a faster MMDs increase in patients treated with the former than with the latter, probably related to the shorter elimination half-life of erenumab. However, this difference was only temporary as migraine frequency was back to baseline the fourth month after discontinuing all the three mAbs.

In our sample, more than one-third of $\mathrm{CM}$ subjects still benefitted from halving of attacks after three months of discontinuation. This represents an important issue as these patients had been very disabled, with at least three failed preventives, and felt relief from their migraine for the first time. No effectiveness or disability parameter per se can fully show the benefit in such resistant patients after taking a treatment capable for the first time to improve their quality of life even if mildly. ${ }^{20}$ One could anticipate that these patients assume to encounter a worsening of their headache rapidly following the pre-imposed stop of three months (nocebo effect). In this line, we could also have expected a rebound increase in MMDs derived at least in part from upregulation of the CGRP receptors during the treatment period. ${ }^{21}$

Beyond analyzing the mere increase of MMDs during the 3-month suspension and the reduction of the percentage of patients with $\geq 50 \% \mathrm{RR}$, the data collected in our 154 patients have other aspects worth highlighting. About $80 \%$ of the 84 baseline CM patients reverted to EM at the end of the 12-month mAbs 
anti-CGRP treatment. Less than half of these patients relapsed to CM up \% at F-UP-3. Moreover, of the patients who had ceased MO (around $80 \%$ ) after one year of treatment, only $1 / 3$ returned to overuse acute medication after the $3^{\text {rd }}$ month of discontinuation (figure 4). One interpretation of these long-term beneficial effects is that weaning the brain from migraine pain by acting in the periphery may have central effects resetting the system to a lower pain load. In support of this hypothesis, in a mouse model of chronic migraine, repeated nitroglycerin (NTG) administration significantly increased the number of CGRP-R and Pituitary adenylate-cyclase activating polypeptide (PACAP)-R neurons in trigeminal ganglion ${ }^{22}$. In this line, a functional magnetic resonance study observed a decreased activation of different structures of the pain network in patients with a positive response to erenumab administration ${ }^{23}$. To note all studies addressing mAbs antiCGRP withdrawal reported a load of MMDs after three months of interruption lower than baseline. One could also speculate that the persisting beneficial effect can derive from the reversal of migraine-driven vicious circles affecting different aspects of a migraineur's life, e.g. lifestyle and psychosocial situations. Once migraine attacks have decreased, patients can enjoy more likely a healthier lifestyle, being less impacted by psychosocial stress. ${ }^{24}$ Interestingly, a longer-lasting benefit (50\%RR at F-UP visits) was not related to clinical baseline characteristics (migraine frequency and disability) or the type of anti-CGRP or CGRP receptor antibody, but to the extent of the beneficial effect obtained in the one-year treatment (last month MMDs and RR, table2 and Figure 3), suggesting that the more effective is the therapy, the more incisive and longer is the impact on migraine.

However, to understand if a disease-modifying effect exists, it would be necessary to observe the clinical course of patients withdrawing the therapy for longer than five half-lives. Unfortunately, this cannot be currently achieved in a real-life observational study.

Our and other evidence from real-life settings may help answer some questions about mAbs anti-CGRP discontinuation and its effect. Our data demonstrated that their therapeutic effect does not outlast after discontinuation in most patients even after a long-term, i.e., one-year, treatment. We know that the half-life of mAbs varies from 26 to 32 days ${ }^{25}$, with their plasma concentration reducing exponentially in the following weeks till reaching one-eights of their initial concentration after 3 months of discontinuation. ${ }^{26}$ Our and other real-world evidence (RWE) translate the pharmacokinetic properties of these drugs in clinical terms as the effectiveness in terms of MMDs reduction and percentages of patients with $50 \%$ RR in most HFEM and CM patients already decreases after one month and gradually and consistently throughout the 3-month discontinuation.

Accordingly, these should not be considered disease-modifying treatments, at least after a one-year administration, as far as we know, but as therapies that have to be continued to maintain their effectiveness, at least in most disabled patients. Nevertheless, some benefits persisted in our and other $\mathrm{RWE}$, especially in $\mathrm{CM}$ and $\mathrm{MO}$ patients, even considering that these are often subjects with a long disease history and many preventives' failures. 
Our data seem not to support the appropriateness of treatment discontinuation after 12 months and a fixed interruption of 3 months in all patients undergoing mAbs anti-CGRP treatment. Most patients of our sample rapidly returned eligible to mAbs anti-CGRP. More practically, since many CM patients with a very high frequency presented at least 10 MMDs and severe disability (60 points at HIT-6 score) at the end of the first month of interruption, one could wonder if it is appropriate to undergo discontinuation after one year of treatment.

Chronic migraine is a very disabling condition, often comorbid with other disorders ${ }^{27}$, deeply affecting patients' emotional and cognitive spheres and different life aspects, including their occupation and career progression, partnership, family planning, and parenting, with dramatic social disadvantage ${ }^{28}$. So that, it is inadequate to impose an interruption, even if of only one month, of the therapy that most of these patients believe is the first effective if we do not have enough data in support. Also, since we observed a relapse to $\mathrm{CM}$ and MO at F-UP3 in less than half of patients treated for one year, we may wonder if a more extended treatment regimen would exert a more significant persistent effect after discontinuation.

In HFEM patients, our findings show contrasting approaches. If, on the one hand, the wearing-off effect seemed more prominent than in CM (figure 1), on the other hand, because of their lower baseline MMDs, HFEM subjects become eligible again to $\mathrm{MAb}$ anti-CGRP in a lower proportion than CM at the F-UP times (figure 2). Nevertheless, at F-UP2, more than half of HFEM patients were again eligible. So that, in HFEM patients, discontinuation could be appropriate to verify when attacks' frequency again reaches the threshold of high-frequency migraine, making necessary to undergo a preventive treatment,

Long-term studies have demonstrated the safety of erenumab in patients treated up to 5 years ${ }^{29}$. Thus medicine regulation agencies based their decisions on discontinuation mainly on cost-saving. Even if RCTs remain the most appropriate way of evaluating the efficacy of therapeutic interventions, real-life studies have become increasingly important in the scientific world in recent years. Although their numerous limitations, they have the advantage of better representing the population we usually have to deal with in our everyday clinical practice. Different RWE about mAbs has provided several helpful data to improve migraine patients' management with these new treatments. ${ }^{8,9,30}$ We have to consider real-life experiences to improve our clinical practice, having the possibility to choose the best management beyond the a priori imposed rules.

Future studies are necessary to explore if any clinical characteristics influence the outcome of mAb antiCGRP discontinuation to individuate subgroups of patients that can stand therapy withdrawal with lower consequences.

\section{Abbreviations}

AIFA =Agenzia Italiana del Farmaco; $\mathrm{Cl}=$ confidence intervals; $\mathrm{CGRP}=$ calcitonin gene-related peptide; $\mathrm{CM}=$ chronic migraine; $\mathrm{EM}$ = episodic migraine, $\mathrm{F}_{-} \mathrm{UP}=$ follow-up; $\mathrm{HFEM}$ = high frequency episodic migraine; HIT-6 = Headache Impact Test 6 items; MMDs = Monthly Migraine Days; mAb= monoclonal antibody; 
MO= medication overuse; $\mathrm{MPI}=$ Monthly Painkillers Intake; NRS =Numerical Rating Scale; MIDAS = Migraine Disability Assessment ; RCT =randomized controlled trials; RR = response rate; RWE = real-world evidence

\section{Declarations}

Ethics approval and consent to participate All patients provided written informed consent. The EARLY study received approval no.19/26 from the IRCCS San Raffaele Pisana Institutional Review Board, while the GARLIT study was approved by the Campus Bio-Medico University Ethical Committee n.30/20. The other local Institutional Review Boards mutually recognized the approvals. The GARLIT study has been registered at the Italian Medicines Agency (Agenzia Italiana del Farmaco, AIFA) and at ClinicalTrials.gov NCT04803513.Consent for publication Not applicable

Availability of data and materials Anonymized data will be shared by request from any qualified investigator.

\section{Competing interests}

Fabrizio Vernieri received travel grants, honoraria for advisory boards, speaker panels, or clinical investigation studies from Allergan, Amgen, Angelini, Eli-Lilly, Lundbeck, Novartis, and Teva.

Roberta Messina received honoraria as speaker from Novartis, Eli Lilly, and Teva.

Bruno Colombo received travel grants, honoraria for advisory boards, speaker panels, or clinical investigation studies from Novartis, Teva, Eli-Lilly Lusofarmaco

Paola Torelli received travel grant, honoraria as a speaker, or for participating in advisory boards from Novartis, Teva, Eli Lilly, and Allergan.

Sabina Cevoli received travel grants, honoraria for advisory boards, speaker panels or clinical investigation studies from Novartis, Teva, Lilly, Allergan, Ibsa, Amgen and Lundbeck.

Valentina Favoni received honoraria as speaker or for participating in advisory boards from Ely-Lilly, Novartis and Teva.

Florindo d'Onofrio receivedgrants and honoraria from Lilly, Teva, Novartis, Neopharmed.

Gabriella Egeo received travel grants and honoraria from Eli-Lilly, Novartis, New Penta and Ecupharma.

Renata Rao receivedhonoraria for speaker panels from Teva, Lilly, Novartis.

Massimo Filippi is the Editor-in-Chief of the Journal of Neurology; received compensation for consulting services and/or speaking activities from Bayer, Biogen Idec, Merck-Serono, Novartis, Roche, Sanofi Genzyme, Takeda, and Teva Pharmaceutical Industries; and receives research support from Biogen Idec, 
Merck-Serono, Novartis, Roche, Teva Pharmaceutical Industries, Italian Ministry of Health, Fondazione Italiana Sclerosi Multipla, and AriSLA (Fondazione Italiana di Ricerca per la SLA).

Piero Barbanti received travel grants, honoraria for advisory boards, speaker panels or clinical investigation studies from Alder, Allergan, Angelini, Bayer, ElectroCore, Eli-Lilly, GSK, Lusofarmaco, MSD, Novartis, Stx-Med, Teva, Visufarma, Zambon.

Claudia Altamura received travel grants and honoraria from Novartis, Eli Lilly, Lusofarmaco, Laborest, Allergan, Almirall.

Nicoletta Brunelli, Simone Quintana and Carmelina Maria Costa have nothing to disclose.

Funding The study costs were covered by Campus Bio-Medico University

Authors' contributions FV and PB designed the study, FV, SC, PB, and CA drafted the manuscript; CA carried out data analysis; NB, RM, CMC, SQ, FF, G E, RR, and VF performed data collection, PT, BC, MF revised the manuscript.

\section{References}

1. Edvinsson L, Haanes KA, Warfvinge K, Krause DiN. CGRP as the target of new migraine therapies Successful translation from bench to clinic. Vol. 14, Nature Reviews Neurology. 2018. p. 338-50.

2. Goadsby PJ, Uwe R, Hallstrom Y, Gregor B, Bonner JH, Zhang F, et al. A controlled trial of erenumab for episodic migraine. N Engl J Med. 2017 Nov 30;377(22):2123-32.

3. Tepper S, Ashina M, Reuter U, Brandes JL, Doležil D, Silberstein S, et al. Safety and efficacy of erenumab for preventive treatment of chronic migraine: a randomised, double-blind, placebo-controlled phase 2 trial. Lancet Neurol. 2017 Jun 1;16(6):425-34.

4. Stauffer VL, Dodick DW, Zhang Q, Carter JN, Ailani J, Conley RR. Evaluation of galcanezumab for the prevention of episodic migraine: The EVOLVE-1 randomized clinical trial. JAMA Neurol. 2018 Sep 1;75(9):1080-8.

5. Detke HC, Goadsby PJ, Wang S, Friedman DI, Selzler KJ, Aurora SK. Galcanezumab in chronic migraine: The randomized, double-blind, placebo-controlled REGAIN study. Neurology. 2018;91(24):E2211-21.

6. Silberstein SD, Dodick DW, Bigal ME, Yeung PP, Goadsby PJ, Blankenbiller T, et al. Fremanezumab for the Preventive Treatment of Chronic Migraine. N Engl J Med. 2017 Nov 30;377(22):2113-22.

7. Dodick DW, Silberstein SD, Bigal ME, Yeung PP, Goadsby PJ, Blankenbiller T, et al. Effect of Fremanezumab compared with placebo for prevention of episodic migraine a randomized clinical trial. JAMA - J Am Med Assoc. 2018 May 15;319(19):1999-2008. 
8. Barbanti P, Aurilia C, Cevoli S, Egeo G, Fofi L, Messina R, et al. Long-term (48 weeks) effectiveness, safety, and tolerability of erenumab in the prevention of high-frequency episodic and chronic migraine in a real world: Results of the EARLY 2 study. Headache. 2021 Jul 26;

9. Vernieri F, Altamura C, Brunelli N, Costa CM, Aurilia C, Egeo G, et al. Galcanezumab for the prevention of high frequency episodic and chronic migraine in real life in Italy: a multicenter prospective cohort study (the GARLIT study). J Headache Pain. 2021 May 3;22(1):35.

10. Headache Classification Committee of the International Headache Society (IHS) The International Classification of Headache Disorders, 3rd edition. Cephalalgia. 2018 Jan 25;38(1):1-211.

11. Sacco S, Bendtsen L, Ashina M, Reuter U, Terwindt G, Mitsikostas DD, et al. European headache federation guideline on the use of monoclonal antibodies acting on the calcitonin gene related peptide or its receptor for migraine prevention. J Headache Pain. 2019 Jan 16;20(1).

12. The American Headache Society Position Statement On Integrating New Migraine Treatments Into Clinical Practice. Headache. 2019 Jan 1;59(1):1-18.

13. Houts CR, Wirth RJ, McGinley JS, Gwaltney C, Kassel E, Snapinn S, et al. Content Validity of HIT-6 as a Measure of Headache Impact in People With Migraine: A Narrative Review. Headache. 2020 Jan $1 ; 60(1): 28-39$.

14. Raffaelli B, Terhart M, Overeem LH, Mecklenburg J, Neeb L, Steinicke M, et al. Migraine evolution after the cessation of CGRP(-receptor) antibody prophylaxis: a prospective, longitudinal cohort study. Cephalalgia. 2021 Sep 27;033310242110466.

15. Raffaelli B, Mussetto V, Israel H, Neeb L, Reuter U. Erenumab and galcanezumab in chronic migraine prevention: Effects after treatment termination. J Headache Pain. 2019 Jun 3;20(1).

16. De Matteis E, Affaitati G, Frattale I, Caponnetto V, Pistoia F, Giamberardino MA, et al. Early outcomes of migraine after erenumab discontinuation: data from a real-life setting. Neurol Sci. 2021 Aug 1;42(8):3297-303.

17. Schiano di Cola F, Caratozzolo S, Venturelli E, Balducci U, Sidoti V, Pari E, et al. Erenumab Discontinuation Following 12-Month Treatment: A Multicentric Observational Real-Life Study. Neurol Clin Pract. 2021 Jul 1;10.1212/CPJ.0000000000001112.

18. Gantenbein AR, Agosti R, Gobbi C, Flügel D, Schankin CJ, Viceic D, et al. Impact on monthly migraine days of discontinuing anti-CGRP antibodies after one year of treatment - a real-life cohort study. Cephalalgia. 2021 May 17;033310242110146.

19. Kuruppu DK, North JM, Kovacik AJ, Dong Y, Pearlman EM, Hutchinson SL. Onset, Maintenance, and Cessation of Effect of Galcanezumab for Prevention of Migraine: A Narrative Review of Three Randomized Placebo-Controlled Trials. Adv Ther. 2021 Mar 1;38(3):1614-26. 
20. Altamura C, Cevoli S, Brunelli N, Aurilia C, Fofi L, Egeo G, et al. When should we consider chronic patients as non-responders to monoclonal antibodies targeting the CGRP pathway? J Neurol. 2021;

21. Tringali G, Vollono C, Calabresi P, Navarra P. A proof-of-concept study on CGRP plasma levels of migraineurs during a 6-month treatment with ERENUMAB. J Headache Pain. 2020 Dec 1;21(1).

22. Guo Z, Czerpaniak K, Zhang J, Cao YQ. Increase in trigeminal ganglion neurons that respond to both calcitonin gene-related peptide and pituitary adenylate cyclase-activating polypeptide in mouse models of chronic migraine and posttraumatic headache. Pain. 2021 May 1;162(5):1483-99.

23. Ziegeler C, Mehnert J, Asmussen K, May A. Central effects of erenumab in migraine patients: An event-related functional imaging study. Neurology. 2020 Sep 11;95(20):e2794-802.

24. Yin JH, Lin YK, Yang CP, Liang CS, Lee JT, Lee MS, et al. Prevalence and association of lifestyle and medical-, psychiatric-, and pain-related comorbidities in patients with migraine: A cross-sectional study. Headache. 2021 May 1;61(5):715-26.

25. Kielbasa W, Helton DL. A new era for migraine: Pharmacokinetic and pharmacodynamic insights into monoclonal antibodies with a focus on galcanezumab, an anti-CGRP antibody. Vol. 39, Cephalalgia. Cephalalgia; 2019. p. 1284-97.

26. Monteith D, Collins EC, Vandermeulen C, Van Hecken A, Raddad E, Scherer JC, et al. Safety, tolerability, pharmacokinetics, and pharmacodynamics of the CGRP binding monoclonal antibody LY2951742 (galcanezumab) in healthy volunteers. Front Pharmacol. 2017 Oct 17;8(OCT).

27. Altamura C, Corbelli I, de Tommaso M, Di Lorenzo C, Di Lorenzo G, Di Renzo A, et al. Pathophysiological Bases of Comorbidity in Migraine. Vol. 15, Frontiers in Human Neuroscience. 2021. p. 640574.

28. Ashina M, Katsarava Z, Do TP, Buse DC, Pozo-Rosich P, Özge A, et al. Migraine: epidemiology and systems of care. Lancet. 2021 Mar 25;

29. Ashina M, Goadsby PJ, Reuter U, Silberstein S, Dodick DW, Xue F, et al. Long-term efficacy and safety of erenumab in migraine prevention: Results from a 5-year, open-label treatment phase of a randomized clinical trial. Eur J Neurol. 2021 May 1;28(5):1716-25.

30. Lambru G, Hill B, Murphy M, Tylova I, Andreou AP. A prospective real-world analysis of erenumab in refractory chronic migraine. J Headache Pain. 2020 Jun 1;21(1).

\section{Tables}

Table 1 Demographical profiles and clinical variables at evaluation times in HFEM and CM patients. 


\begin{tabular}{|c|c|c|}
\hline & HFEM $(n=47)$ & CM $(n=107)$ \\
\hline Age years, mean (SD) & $48.9(11.2)$ & $49.4(11.2)$ \\
\hline Sex \% (n females) & $78.7(37)$ & $69.2(74)$ \\
\hline BMI kg/m², median $\left(25^{\text {th }}, 75^{\text {th }}\right)$ & $22.00(20.43,23.63)$ & $23.20(22.00,25.70)$ \\
\hline MO \% (n) & $9.7(10)$ & $90.3(93)$ \\
\hline Treatment \% (n Erenumab) & $51.1(24)$ & $62.6(67)$ \\
\hline \multicolumn{3}{|l|}{ MMDs median $\left(25^{\text {th }}, 75^{\text {th }}\right)$} \\
\hline Baseline & $11.0(10.0,13.75)$ & $20.0(16.0,30.0)$ \\
\hline last month & $6.0(4.0,9.0)$ & $9.0(5.7,14.3)$ \\
\hline follow-up 1 month & $9.0(8.0,11.0)$ & $14.5(10.0,18.0)$ \\
\hline follow-up 2 month & $10.5(9.2,11.7)$ & $16.0(10.7,20.0)$ \\
\hline follow-up 3 month & $10.5(9.0-13.5)$ & $15.0(10.7,22.0)$ \\
\hline \multicolumn{3}{|l|}{ RR median $\left(25^{\text {th }}, 75^{\text {th }}\right)$} \\
\hline last month & $-63.6(-84.7,-60.0)$ & $-61.1(37.3)$ \\
\hline follow-up 1 month & $-42.9(-87.9,-42.5)$ & $-46.6(54.2)$ \\
\hline follow-up 2 month & $-28.6(-71.5,2-20.5)$ & $-40.0(45.6)$ \\
\hline follow-up 3 month & $-14.3(-65.9,-10.3)$ & $-30.0(48.3)$ \\
\hline last month - follow up 3 difference & $-47.7(-79.5,-17.0)$ & $-25.5(-47.1,-3.3)$ \\
\hline \multicolumn{3}{|l|}{ MPI median $\left(25^{\text {th }}, 75^{\text {th }}\right)$} \\
\hline Baseline & $12.0(10.5,14.5)$ & $21.0(15.0,32.0)$ \\
\hline last month & $4.0(2.0,7.0)$ & $7.0(4.0,13.0)$ \\
\hline follow-up 1 month & $6.0(2.5,11.0)$ & $8.0(5.0,15.0)$ \\
\hline follow-up 2 month & $9.0(5.5,12.5)$ & $12.0(8.0,18.0)$ \\
\hline follow-up 3 month & $10.0(6.0,13.5)$ & $15.0(9.0,22.0)$ \\
\hline \multicolumn{3}{|l|}{ NRS median $\left(25^{\text {th }}, 75^{\text {th }}\right)$} \\
\hline Baseline & $7.5(7.0,8.0)$ & $7.0(7.0,8.0)$ \\
\hline last month & $6.0(4.0,6.2)$ & $5.0(4.0,7.0)$ \\
\hline follow-up 1 month & $6.0(4.7,7.0)$ & $6.0(4.0,7.0)$ \\
\hline
\end{tabular}




\begin{tabular}{|lll|}
\hline follow-up 2 month & $6.0(4.7,7.0)$ & $6.0(5.0,7.0)$ \\
\hline follow-up 3 month & $7.0(5.0,7.0)$ & $7.0(5.0,8.0)$ \\
\hline Hit-6 median $\left(25^{\text {th }}, 75^{\text {th }}\right)[\mathrm{n}]$ & & \\
\hline Baseline & $66.5(64.7,70.0)[33]$ & $67.0(64.0,72.0)[83]$ \\
\hline last month & $51.0(41.5,60.0)[24]$ & $55.0(50.0,62.0)[57]$ \\
\hline follow-up 1 month & $56.0(31.5,62.3)[20]$ & $60.0(53.0,66.0)[51]$ \\
\hline follow-up 2 month & $61.5(51.5,65.0)[22]$ & $62.0(53.5,66.5)[51]$ \\
\hline follow-up 3 month & $60.5(48.3,65.3)[37]$ & $63.0(51.0,68.0)[75]$ \\
\hline
\end{tabular}

Table 2 Demographical profiles and clinical variables at evaluation times in patients with 50\%RR at F-UP1 and F-UP3 (i.e. long-lasting responders) compared with non-lasting-responders. 


\begin{tabular}{|c|c|c|c|c|c|c|}
\hline & $\begin{array}{l}\text { F-UP1 } \\
50 \% R R \\
(n=70)\end{array}$ & $\begin{array}{l}\text { F-UP1 no } \\
50 \% R R \\
(n=84)\end{array}$ & p & $\begin{array}{l}\text { FUP-3 } \\
50 \% R R \\
(n=51)\end{array}$ & $\begin{array}{l}\text { F-UP3 no } \\
\% \text { RR } \\
(n=103)\end{array}$ & p \\
\hline $\begin{array}{l}\text { Age years, mean } \\
\text { (SD) }\end{array}$ & $49.1(10.8)$ & $49.7(11.4)$ & .746 & $47.7(11.6)$ & $49.8(11.0)$ & .298 \\
\hline Sex \% (n females) & $71.4(50)$ & $75.3(61)$ & 1.000 & 76.4 (39) & $69.9(72)$ & .313 \\
\hline $\begin{array}{l}\text { BMI kg/m², median } \\
\left(25^{\text {th }}, 75^{\text {th }}\right)\end{array}$ & $\begin{array}{l}22.80(20.85, \\
25.92)\end{array}$ & $\begin{array}{l}22.60 \\
(21.00 \\
24.50)\end{array}$ & .752 & $\begin{array}{l}23.40 \\
(21.30 \\
26.40)\end{array}$ & $\begin{array}{l}22.07 \\
(20.93 \\
24.00)\end{array}$ & .110 \\
\hline CM \% (n) & $67.1(47)$ & $71.4(60)$ & .595 & 74.5 (38) & $66.9(69)$ & .438 \\
\hline Baseline MO \% (n) & $67.1(47)$ & $66.7(56)$ & 1.000 & $76.4(39)$ & $62.1(64)$ & .131 \\
\hline $\begin{array}{l}\text { Treatment \% ( } \mathrm{n} \\
\text { Erenumab) }\end{array}$ & $59.4(41)$ & $61.0(50)$ & .489 & $50.9(26)$ & $63.1(65)$ & .195 \\
\hline \multicolumn{7}{|l|}{$\begin{array}{l}\text { MMDs median } \\
\left(25^{\text {th }}, 75^{\text {th }}\right)\end{array}$} \\
\hline Baseline & $\begin{array}{l}16.0 \\
(12.0,25.0)\end{array}$ & $\begin{array}{l}15.5 \\
(12.8,20.0)\end{array}$ & .928 & $\begin{array}{l}15.0 \\
(12.0,25.0)\end{array}$ & $\begin{array}{l}15.0 \\
(11.0,22.5)\end{array}$ & .545 \\
\hline Last month & $4.0(2.0,6.5)$ & $\begin{array}{l}9.0 \\
(5.0,14.0)\end{array}$ & $<.001$ & $3.5(2.0,7.0)$ & $\begin{array}{l}6.0 \\
(4.0,12.5)\end{array}$ & $<.001$ \\
\hline $\begin{array}{l}\text { Last month RR } \\
\text { median }\left(25^{\text {th }}, 75^{\text {th }}\right)\end{array}$ & $\begin{array}{l}-77.3 \\
(-85.4,-60.0)\end{array}$ & $\begin{array}{l}-41.9 \\
(-63.6,-21.1)\end{array}$ & $<.001$ & $\begin{array}{l}-80.0 \\
(-86.7,-62.5)\end{array}$ & $\begin{array}{l}-56.7 \\
(-72.2,-31.7)\end{array}$ & $<.001$ \\
\hline \multicolumn{7}{|l|}{$\begin{array}{l}\text { MPI median } \\
\left(25^{\text {th }}, 75^{\text {th }}\right)\end{array}$} \\
\hline Baseline & $\begin{array}{l}18.0 \\
(12.0,30.0)\end{array}$ & $\begin{array}{l}18.0 \\
(13.0,29.3)\end{array}$ & .912 & $\begin{array}{l}18.0 \\
(13.0,30.0)\end{array}$ & $\begin{array}{l}15.0 \\
(12.0,30.0)\end{array}$ & .594 \\
\hline Last month & $4.0(2.0,7.0)$ & $\begin{array}{l}9.0 \\
(5.0,13.0)\end{array}$ & $<.001$ & $3.0(1.0,6.0)$ & $\begin{array}{l}8.0 \\
(4.0,12.0)\end{array}$ & $<.001$ \\
\hline \multicolumn{7}{|l|}{$\begin{array}{l}\text { NRS median } \\
\left(25^{\text {th }}, 75^{\text {th }}\right)\end{array}$} \\
\hline Baseline & $7.0(6.0,8.0)$ & $7.0(7.0,8.0)$ & .226 & $\begin{array}{l}7.0 \\
(6.0,8.0)\end{array}$ & $7.0(7.0,8.0)$ & .887 \\
\hline Last month & $5.0(3.0,6.0)$ & $6.0(5.0,7.0)$ & $<.001$ & $4.0(3.0,6.0)$ & $6.0(5.0,7.0)$ & $<.001$ \\
\hline \multicolumn{7}{|l|}{$\begin{array}{l}\text { HIT-6 median } \\
\left(25^{\text {th }}, 75^{\text {th }}\right)\end{array}$} \\
\hline Baseline & $\begin{array}{l}69.0 \\
(64.0,72.5)\end{array}$ & $\begin{array}{l}66.0 \\
(65.0,69.0)\end{array}$ & .052 & $\begin{array}{l}69.0 \\
(64.0,73.0)\end{array}$ & $\begin{array}{l}66.0 \\
(64.0,70.0)\end{array}$ & .075 \\
\hline Last month & 52.0 & 58.0 & .001 & 44.0 & 57.0 & $<.001$ \\
\hline
\end{tabular}




\section{Figures}

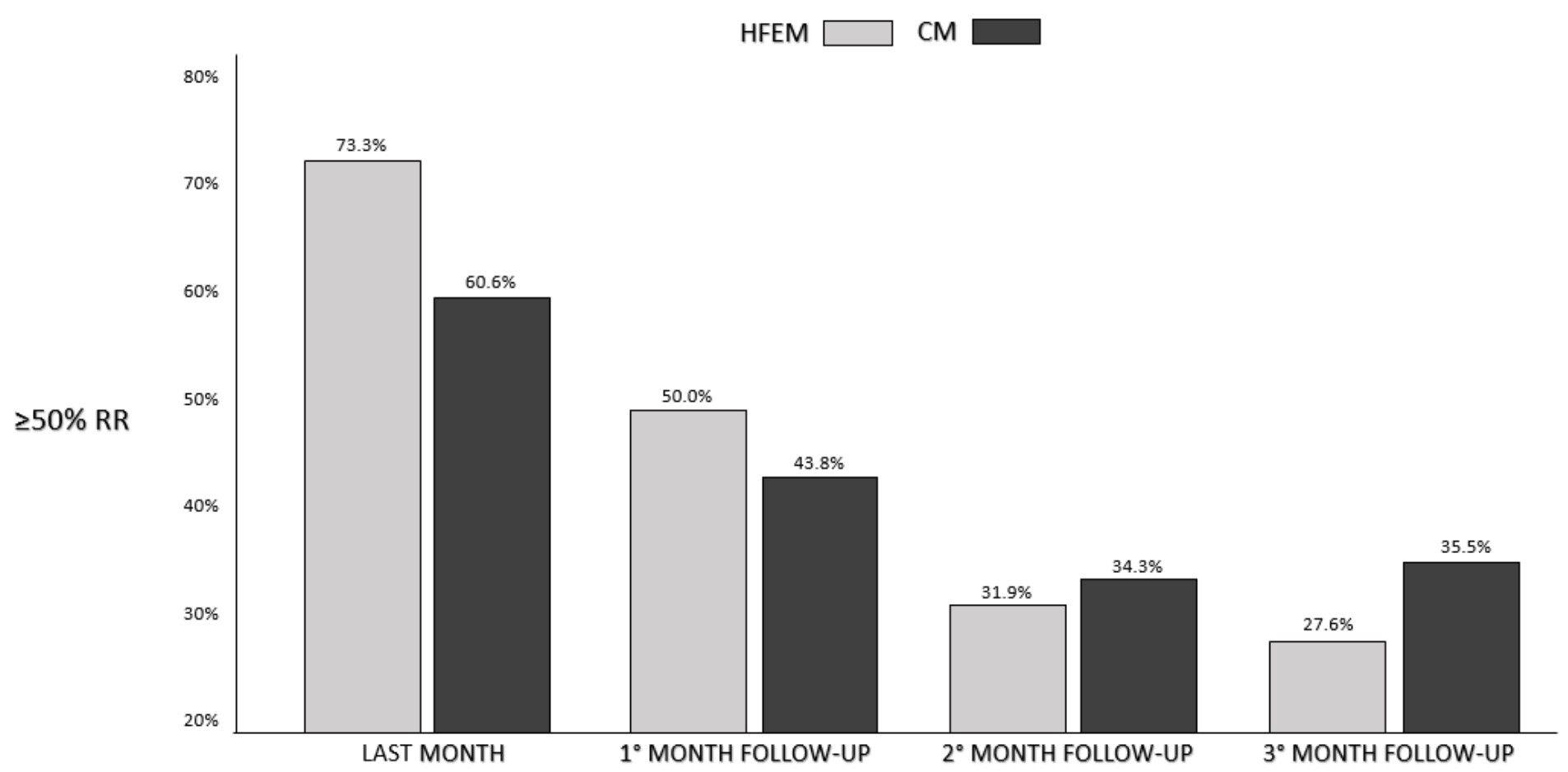

\section{Figure 1}

The $50 \%$ response rates at the last month of therapy with mAbs anti-CGRP pathway and at the threemonths of discontinuation in CM and HFEM patients. 


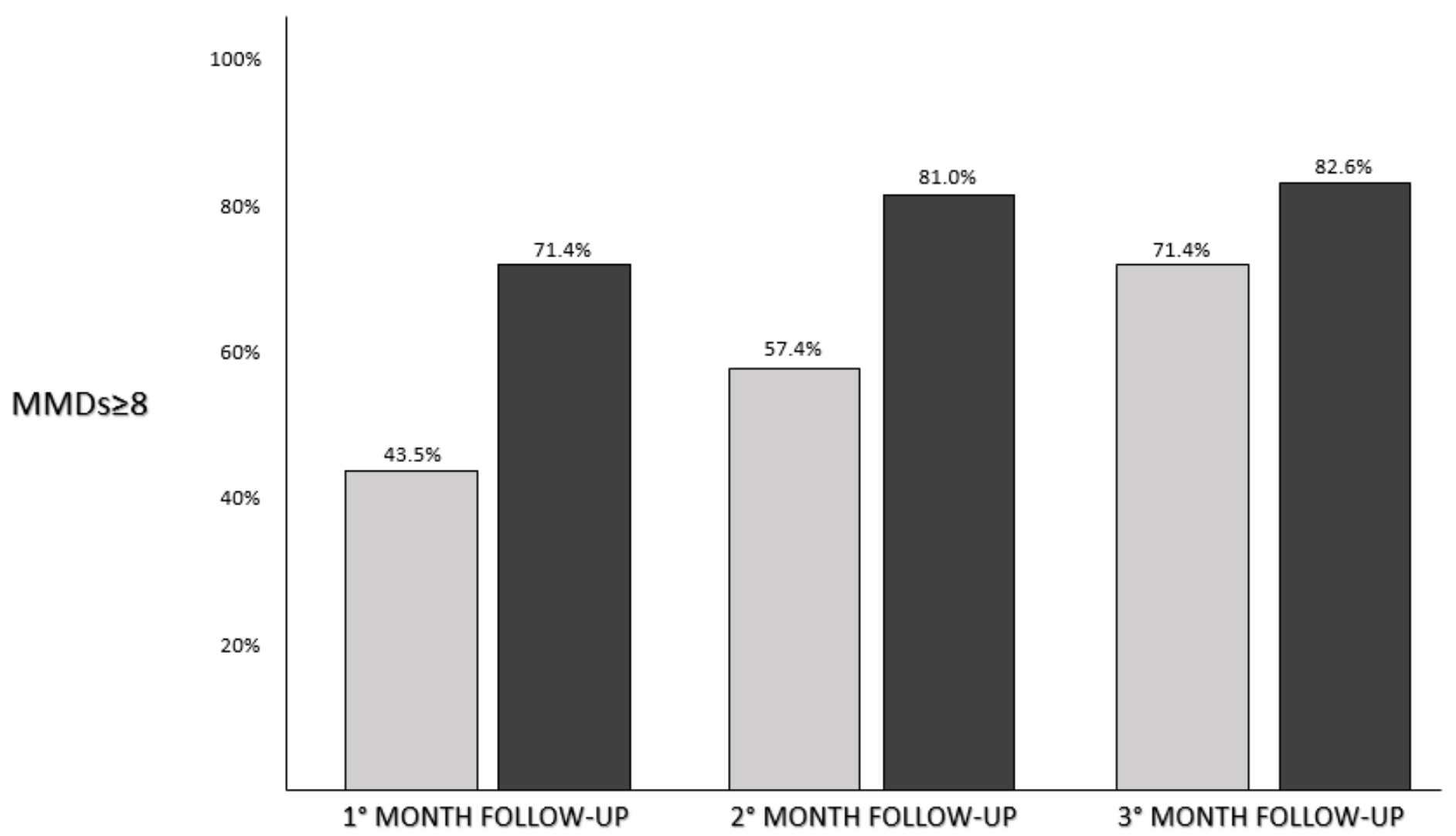

Figure 2

The percentage of patients having at least 8 MMDs in the three months of mAbs anti-CGRP pathway discontinuation in CM and HFEM patients.

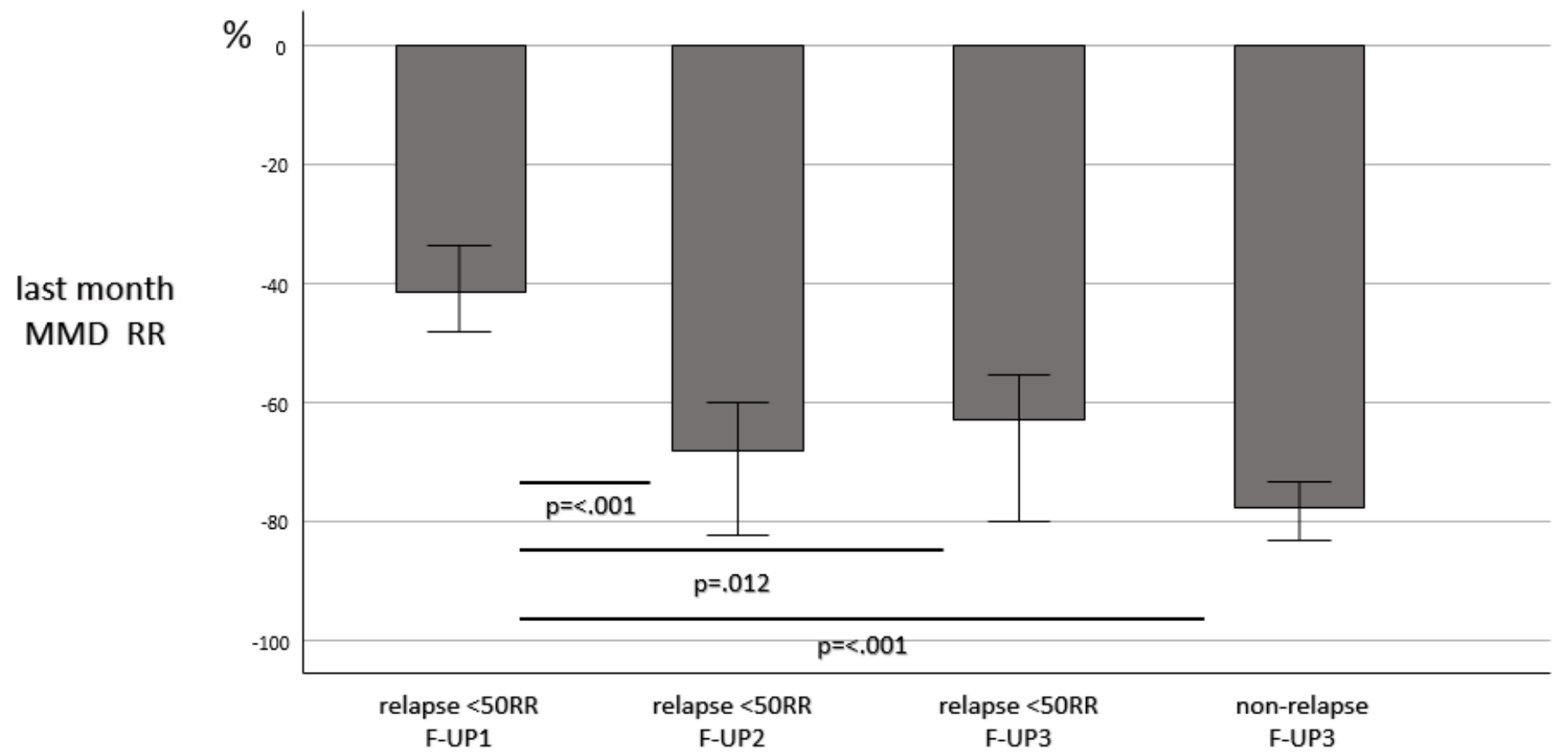




\section{Figure 3}

The median value (95\% $\mathrm{Cl}$ bars) of MMDs percentual reduction (RR) in the last month of therapy in patients relapsing to $<50 \%$ RR response at F-UP1, F-UP2, F-UP3, and patients still responding at F-UP3 (Mann-Whitney U test)

A

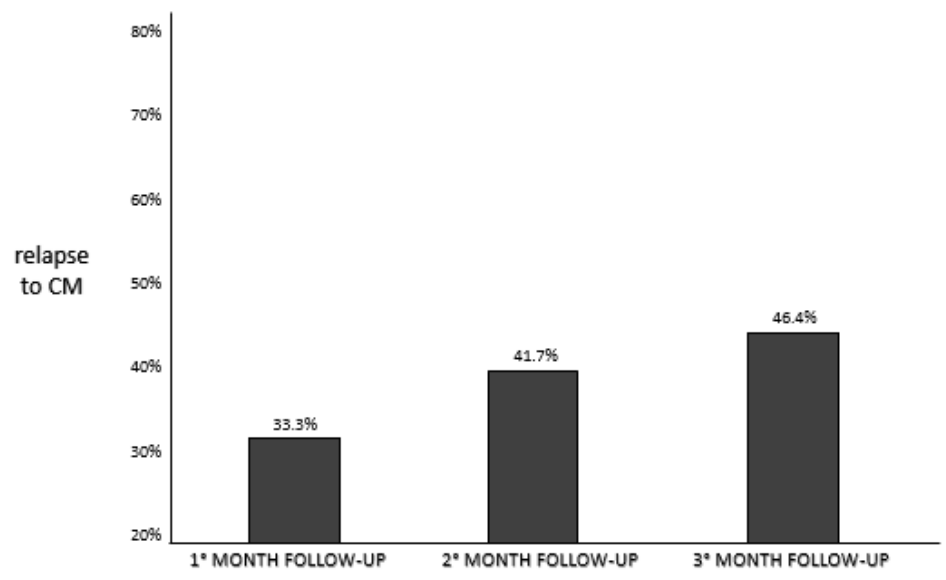

B

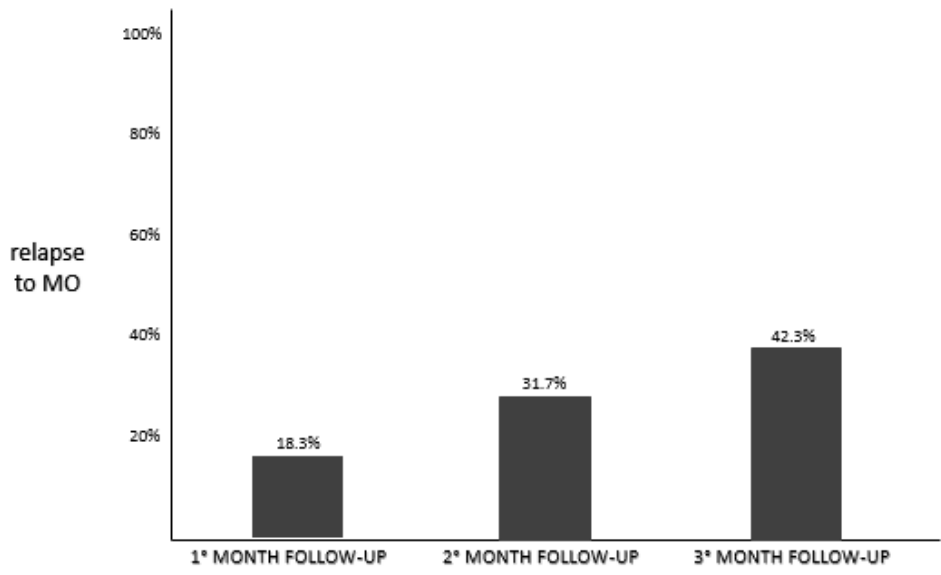

Figure 4

The percentage of patients relapsing to $\mathrm{CM}(\mathrm{A})$ and $\mathrm{MO}(\mathrm{B}) \mathrm{MMDs}$ in the three months of mAbs antiCGRP pathway discontinuation.

\section{Supplementary Files}

This is a list of supplementary files associated with this preprint. Click to download.

- strobechecklistJHP.doc 